\title{
EL CONSENSO DE LOS ANALISTAS FINANCIEROS ANTES Y DURANTE \\ LA CRISIS FINANCIERA: UNA EVIDENCIA EN EL MERCADO CONTINUO' ${ }^{1}$
}

\author{
Author: Ignacio Cervera Conte \\ Profesor del Departamento de Gestión Financiera \\ Facultad de Ciencias Económicas y Empresariales \\ Universidad Pontificia Comillas ${ }^{2}$
}

\section{Resumen}

El presente artículo tiene el objetivo de concretar el efecto del consenso de los analistas financieros en el Mercado Continuo español en los periodos 2002-2007 y 2008-2009 con el fin de determinar su incidencia en la etapa de la crisis financiera.

Este artículo concluye que el consenso de los analistas financieros tiene una influencia cuantitativamente pequeña pero significativa en la volatilidad de los

\footnotetext{
${ }^{1}$ El autor agradece la ayuda prestada por los profesores Teresa Corzo y Ricardo Gimeno y los valiosos comentarios de la profesora Margarita Prat.

${ }^{2}$ icervera@icade.comillas.edu
} 
mercados españoles. La incertidumbre asociada a la crisis hace que la influencia del consenso aumente.

Palabras clave: analista financiero; estimaciones de consenso; precio objetivo; previsión de ingresos; recomendación analista.

Analysts consensus before and during the financial crisis: evidence on the spanish market (mercado continuo)

\begin{abstract}
The goal of this article is to determine the effects of financial analysts' consensus in the Spanish Stock Market (Mercado Continuo) in different periods (2002-2007 and 2008-2009), specifically its influence during the financial crisis.

This article concludes that the effects of financial analysts' consensus is modest but statistically significant in the Spanish Mercado Continuo, and is also stronger during the financial crisis.
\end{abstract}

Key words: financial analyst; consensus estimates; target price; earning forecast; analyst recommendation.

\title{
1. INTRODUCCIÓN
}

El objetivo de este trabajo es ver el rol que los analistas financieros tienen en los mercados bursátiles: Se pretende ver qué influencia efectiva tienen, cómo se interpreta esa influencia y qué cambio ha experimentado esta influencia con la aparición de la crisis financiera.

Los mercados financieros secundarios en general y los mercados bursátiles en particular permiten a los agentes económicos el intercambio de activos financieros previamente emitidos. Estos mercados financieros están afectados por las fuerzas de oferta y demanda de dichos activos que acaban fijando el precio de los mismos. Los inversores buscan en estos mercados los activos financieros que mejor se adecúen a sus necesidades financieras, a sus perfiles de riesgo y sobre todo a sus expectativas. Por esta última razón, se dice que los mercados financieros reflejan en los precios de los activos financieros las expectativas de los agentes que intervienen en ellos. 
En este contexto, los inversores tienen diversas fuentes de información disponibles para ellos. Hirst et al. (1995) consideran que los informes de los analistas son los que más influencia tienen en la toma de decisiones sobre inversiones. Los analistas financieros son importantes intermediarios de la información acerca de las empresas cotizadas. Sus estimaciones y recomendaciones ejercen una influencia en las expectativas de los mercados y, por ello, son utilizadas muchas veces como una medida representativa de las expectativas no observables de los agentes que intervienen en los mercados financieros (Schipper, 1991; Abarbanell et al., 1995; Ramnath et al., 2008).

Los analistas financieros han sido criticados por diversos motivos, especialmente en épocas de crisis bursátiles y financieras. Entre estos motivos se encuentran el sesgo optimista de sus estimaciones (Brav y Lehavy, 2003; Cowen et al., 2006; Kerl y Walter, 2008), la objetividad mermada por conflictos de interés (Gimein, 2002; Cowen et al., 2006), los precios objetivos alejados de la realidad (Morgenson, 2001; Maiello, 2000) y el seguimiento gregario del mercado (Jegadeesh y Kim, 2010).

En el contexto español, en el año 2008 varias firmas que por entonces cotizaban en el Mercado Continuo se quejaban de los comentarios que en sus informes realizaban los analistas de varias instituciones financieras: Jazztel y Zeltia acerca de los analistas de Goldman Sachs, ACS acerca de un analista de Credit Suisse y la AEB (Asociación Española de la Banca) por los análisis de AFI (Analistas Financieros Internacionales) acerca de algunos bancos, por citar algunos ejemplos. La queja surgía por la repercusión negativa que tienen los análisis en las cotizaciones bursátiles.

Sin embargo, resulta contradictorio que un analista no tenga repercusión con sus análisis. Porque los inversores ponen en valor precisamente la información aportada por los analistas, se espera que sus estimaciones tengan repercusión. Si los datos que cuentan para las estimaciones son negativos, éstas serán negativas y por tanto se espera que produzca una reacción negativa; producirá una reacción positiva si los datos que utilizan para sus estimaciones reflejan un futuro positivo para la empresa que analiza.

Desde esta situación resultan oportunas las siguientes cuestiones: ¿Qué influencia efectiva tienen los analistas financieros? ¿Cómo se interpreta esa influencia? ¿Qué cambio ha experimentado esta influencia con la aparición de la crisis financiera? Los siguientes apartados están destinados a responder a estas preguntas.

Para responder a estas preguntas se procede a describir en un primer apartado el trabajo de los analistas visto por la literatura financiera. Después se presenta la metodología utilizada para analizar el efecto del consenso de los analistas en la volatilidad de acciones que cotizan en el Mercado Continuo. En tercer lugar, se presentan los resultados obtenidos. En cuarto lugar, se procede a una interpretación de los resultados. Por último se concluye que aunque la influencia del consenso de los analistas en el Mercado Continuo es pequeño pero significativo, durante la crisis financiera el aumento de la incertidumbre ha hecho que el papel del consenso de los analistas financieros sea algo más intenso. 


\section{EL TRABAJO DE LOS ANALISTAS FINANCIEROS}

En los mercados financieros existen tres tipos de agentes que interactúan continuamente: los directivos de las empresas cuyas acciones cotizan en mercados organizados, los analistas financieros que trabajan fundamentalmente para agencias y sociedades de valores y para los gestores de fondos institucionales de inversión $\mathrm{y}$, finalmente, los inversores.

El analista financiero tiene como papel primordial analizar la situación financiera de la empresa y realizar un diagnóstico que refleje aspectos como la rentabilidad, la liquidez y el riesgo.

Puede desarrollar su labor en una empresa financiera, en una consultoría o como profesional externo que asesora a varias compañías. También puede trabajar en entidades financieras como gestor de créditos y de inversiones o llevando la cartera de valores de los clientes.

El analista analiza los balances, cuentas de resultados e informes anuales que publican las compañías, bien directamente o bien a través del regulador. Elabora también estudios financieros (inversiones, control de costes, rentabilidad...), en los que evalúa los posibles riesgos y valora las diferentes estrategias a seguir. Sigue toda la información financiera que pueda ser relevante para la compañía, desde el seguimiento en los mercados de valores y las cotizaciones de diferentes firmas hasta el estudio de la situación política y económica internacional. El trabajo de los analistas es un proceso que consume tiempo y dinero. Este hecho ha llevado a muchas compañías a subcontratar este servicio a analistas externos.

Según la relación que el analista mantenga con la casa que le contrata, se hablará de analistas del lado de la compra ${ }^{3}$, analistas del lado de la venta ${ }^{4} \mathrm{y}$ analistas independientes.

Los analistas del lado de la venta generalmente trabajan para agencias y sociedades de valores que ofrecen servicios tanto de asesoramiento como de gestión de compra-venta de títulos. Sus informes son utilizados para vender una idea a sus clientes individuales o institucionales. Las recomendaciones y calificaciones de estos analistas van encaminadas a colocar una inversión y son típicamente ofrecidas a los clientes de las agencias y sociedades de valores mencionadas. Los inversores individuales acceden a estos informes abriendo cuentas en sus respectivos intermediarios financieros. Sus informes son generalmente más detallados que los de los analistas del lado de la compra. Un buen informe de un analista del lado de la venta contiene análisis detallados de las ventajas competitivas de la compañía que analiza, información sobre las

\footnotetext{
${ }^{3}$ Buy-side analysts, en la terminología financiera anglosajona.

${ }^{4}$ Sell-side analysts, en la terminología financiera anglosajona.
} 
habilidades gerenciales del equipo directivo y cómo está operando la compañía y están siendo valoradas sus acciones en comparación con grupos de empresas afines y con su industria.

Los analistas del lado de la compra aunque formulan predicciones suelen tener un papel más consultivo (Larran y Rees, 2000); trabajan para grandes compañías institucionales de inversión tales como los fondos de inversión o las compañías de seguros. Ofrecen recomendaciones sobre títulos valores para sus compañías y centran sus investigaciones sobre sectores o valores específicos que son del interés de las compañías de inversión. Sus informes son más breves y son generalmente de uso interno de los directores de los fondos.

Los analistas independientes no son empleados por agencias y sociedades de valores ni por instituciones de inversión. Los analistas independientes buscan emitir informes y otorgar calificaciones insesgadas y objetivas. Reciben una compensación por sus informes bien de las compañías que analizan ${ }^{5}$ o bien de la venta de suscripciones a sus informes ${ }^{6}$. En cualquier caso, los analistas independientes deben clarificar explícitamente la relación que tienen con la compañía que está siendo objeto de su análisis.

Debido a que los analistas del lado de la compra únicamente emiten informes para las instituciones en las que trabajan, este trabajo sólo tendrá en cuenta las opiniones de los analistas del lado de la venta y los analistas independientes.

Escribir informes expresando opiniones es siempre parte del trabajo de los analistas de valores. Tradicionalmente los analistas utilizan los principios del análisis fundamental (Penman, 2010); sin embargo, los análisis técnicos y la valoración del contexto del mercado son también parte de su rutina profesional. En los informes que emiten se ofrecen, para un determinado horizonte temporal, tres tipos de estimaciones (Feldman et al., 2012; Asquith et al., 2005): predicciones de beneficios y dividendos al final del ejercicio que están analizando así como el de los ejercicios siguientes; estimación de un precio objetivo a alcanzar en los próximo doce meses; finalmente ofrecen una recomendación de inversión del tipo comprar/vender/mantener.

Sus fuentes de información se encuentran en los informes públicos (estadísticas y evolución de las macro-magnitudes, de la productividad y del empleo) y en los estados financieros auditados de las compañías, así como en las presentaciones ${ }^{7}$ ofrecidas por los directores financieros, donde los analistas tienen la oportunidad de preguntarles directamente.

Analizan frecuentemente toda una industria o sector, valorando las tendencias de los negocios en dicha industria. Prestan también atención a las nuevas re-

\footnotetext{
${ }^{5}$ Fee-based research, en la terminología financiera anglosajona.

${ }^{6}$ Suscription-based research, en la terminología financiera anglosajona.

${ }^{7}$ Road shows, en la terminología financiera anglosajona.
} 
gulaciones o políticas que puedan afectar al sector, así como a las magnitudes macroeconómicas que puedan afectar a las ganancias de la industria que examinan.

Sobre la base de sus resultados ellos emiten informes y realizan presentaciones, normalmente haciendo recomendaciones para comprar o vender un determinado activo financiero. Es de esperar que un inversor siga la información de los analistas con los que mantiene un contacto profesional más asiduo; pero también es de esperar se fije en el consenso de los analistas que siguen los valores en los que está interesado. El presente trabajo tiene la finalidad de examinar la influencia de este consenso en el mercado.

\section{DISEÑO DE LA INVESTIGACIÓN}

En la mayoría de los trabajos realizados en este sentido, los investigadores consideran globalmente a los analistas mediante una media de las estimaciones de un conjunto de analistas individuales. En este trabajo se va a considerar la globalidad de los analistas que siguen el Mercado Continuo español mediante el consenso elaborado por la casa de datos FactSet, de uso muy extendido en las aplicaciones y publicaciones financieras en lengua castellana.

La escasez de estudios internacionales centrados en la influencia de los analistas en el mercado español y la facilidad de acceso a sus datos hace que el presente artículo se centre en el Mercado Continuo español.

\subsection{Periodo y periodicidad}

El periodo se establece de acuerdo a la disponibilidad de datos de la base FactSet, para obtener el mayor número posible de variables, el mayor número posible de compañías y el periodo de tiempo más largo posible. Si se expande el periodo arrancando desde el año 2000, algunas compañías no estaban cotizando en ese momento; si se expande el periodo más allá del 2009, algunas compañías desaparecerían de la muestra, bien porque se han fusionado con otras o bien porque han dejado de cotizar. Con el periodo elegido se asegura una muestra equilibrada.

Este periodo goza además de la peculiaridad de recoger la fase anterior y la fase de comienzo de la crisis, permitiendo un análisis comparativo de ambas fases. El periodo, que comprende los meses que van desde enero del 2002 hasta diciembre del 2009, comienza con la recuperación de la crisis bursátil producida por el estallido de la burbuja de las empresas punto.com y finaliza en la crisis financiera que comienza en el año 2007 pero cuyos fuertes efectos no comienzan a sentirse hasta 2008 y sigue en el año 2009, como puede observarse en los siguientes gráficos: 
El CONSENSO DE LOS ANALISTAS FINANCIEROS ANTES Y DURANTE LA CRISIS FINANCIERA...

Gráfico 1. Precios de Cierre del Ibex 35

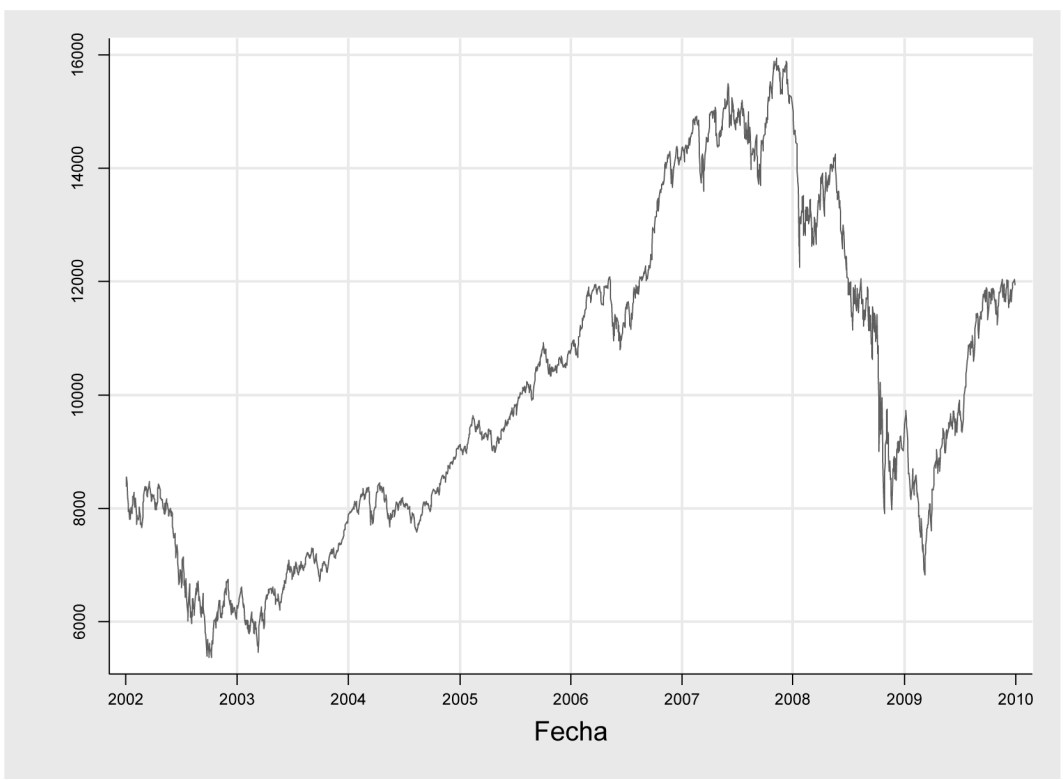

Fuente: elaboración propia a partir de los datos de Infobolsa.

\section{Gráfico 2. Volatilidad del Ibex 35}

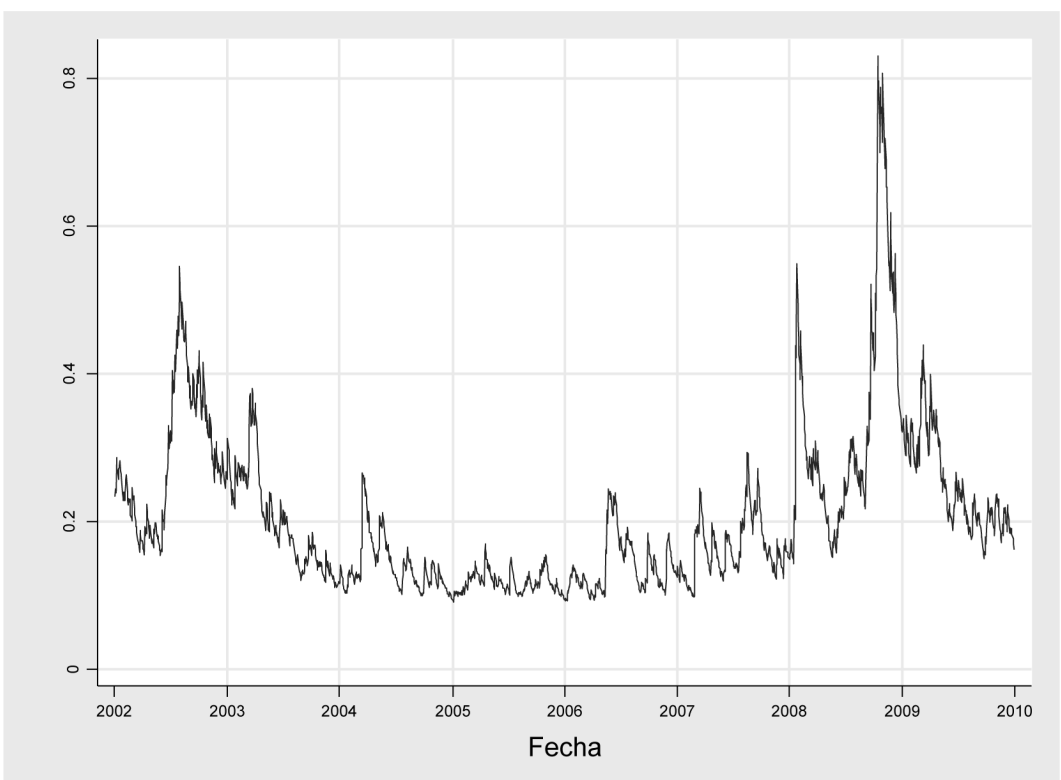

Fuente: elaboración propia a partir de los datos de Infobolsa. 
Ignacio Cervera Conte

Con esta delimitación temporal se puede examinar el impacto de las estimaciones de los analistas antes (2002-2007) y después (2008-2009) de la aparición de la crisis.

\subsection{La muestra}

La investigación se ha realizado a partir de los datos que aportan las siguientes fuentes secundarias:

- Para los datos históricos: Base de datos Infobolsa.

- Para los datos del consenso de los analistas: Base de datos FactSet.

Estos analistas están representados a través de los brokers para quienes trabajan. Por consiguiente, el consenso no está formado por el número de analistas individuales sino por el número de brokers que cubren el seguimiento de los títulos del mercado bursátil. La tabla 1 recoge el número de brokers que cubren el seguimiento de las empresas de la muestra y que constituyen la base para las cifras de consenso:

Tabla 1. Número de brokers de la muestra-Mercado Continuo

\begin{tabular}{|c|c|c|}
\hline Año & Número de brokers & $\begin{array}{c}\text { Número medio de brokers } \\
\text { por empresa }\end{array}$ \\
\hline 2002 & 78 & 19,4 \\
\hline 2003 & 72 & 18,1 \\
\hline 2004 & 76 & 16 \\
\hline 2005 & 68 & 15 \\
\hline 2006 & 72 & 15,3 \\
\hline 2007 & 65 & 15,2 \\
\hline 2008 & 68 & 15,4 \\
\hline 2009 & 62 & 14,7 \\
\hline
\end{tabular}

Fuente: FactSet.

La muestra comprende 50 empresas del Mercado Continuo español, en sus modalidades de contratación Open y Fixing. El periodo de análisis abarca 96 meses que van desde enero 2002 hasta diciembre 2009. Por consiguiente, la muestra está constituida por 4.800 observaciones empresa-mes.

De estas 50 empresas, 20 pertenecían al IBEX 35 el 31 de diciembre de 2009, es decir, un $40 \%$ de las empresas de la muestra. Estas 20 empresas representan el $90,13 \%$ del total de la capitalización de las empresas de la muestra en la misma fecha, como se puede observar en el gráfico 3: 
Gráfico 3. Capitalización de la muestra al 31/12/2009

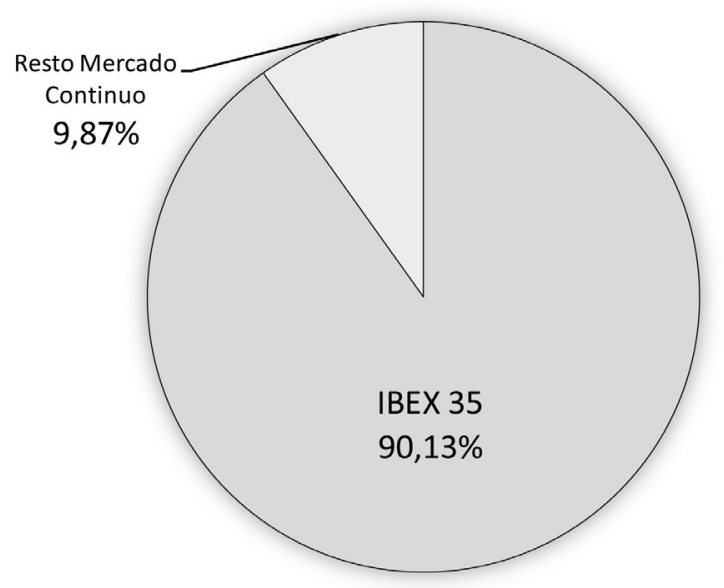

Fuente: elaboración propia a partir de los datos de Infobolsa.

Cuando se compara esta proporción con la proporción que guarda el IBEX 35 con respecto al Mercado Continuo al 31 de diciembre de 2009 se observa que las empresas del índice selectivo español representan el 27,56\% con respecto al total de empresas que cotizan en el Mercado Continuo, y el 72,58\% con respecto a la capitalización alcanzada en dicha fecha, como queda ilustrado en el gráfico 4:

\section{Gráfico 4. Capitalización del Mercado Continuo al 31/12/2009}

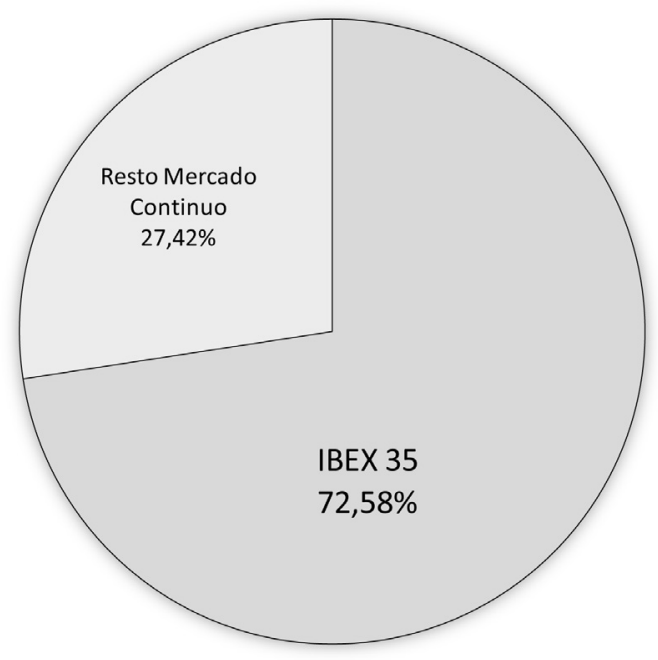

Fuente: elaboración propia a partir de los datos de Infobolsa. 
Estas proporciones de las empresas del IBEX 35 con el total del Mercado Continuo son menores que las mantenidas con el total de la muestra. Las 50 empresas de la muestra representan el 79,13\% de la capitalización del Mercado Continuo al 31 de diciembre de 2009, como ilustra el gráfico 5:

\section{Gráfico 5. Capitalización del Mercado Continuo al 31/12/2009 (incluido muestra)}

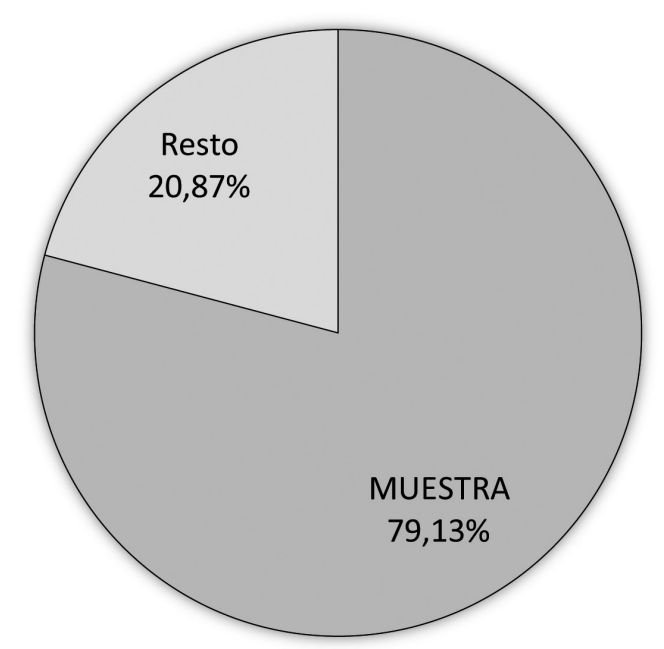

Fuente: elaboración propia a partir de los datos de Infobolsa.

Las que pertenecen al IBEX Medium Cap e IBEX Small Cap representan respectivamente el 76,48\% y 65,10\% de la capitalización de sus correspondientes índices.

La muestra recoge un porcentaje muy alto de la capitalización de cada sector representativo:

- Servicios financieros e inmobiliarios. . . . . . . . . . 82,73\%

- Petróleo y energía . . . . . . . . . . . . . . . . 85,83 \%

- Tecnología y telecomunicaciones ............ 96,66\%

Todas estas cifras evidencian que el Mercado Continuo español queda suficientemente representado en esta muestra.

Como se trata de ver qué influencia ejerce el consenso de los analistas en el mercado, la volatilidad de las rentabilidades de los valores cotizados va a ser la magnitud que va a representar al mercado. La crisis añade incertidumbre a los mercados y la volatilidad es muy sensible a esta incertidumbre. Se trata pues de ver qué influencia ejerce el consenso de los analistas en la volatilidad. 
Las opiniones de los analistas se materializan en los tres grupos de estimaciones mencionados anteriormente: beneficios estimados, precios objetivos y recomendaciones. La literatura financiera dice que las revisiones de las estimaciones son más relevantes que las estimaciones mismas (Ramnath et al., 2008).

\subsection{El modelo}

Para cuantificar la influencia del consenso de los analistas en la volatilidad del mercado se ha hecho uso de un tipo de modelo que tiene en cuenta las siguientes circunstancias que pueden sesgar los resultados:

a) la volatilidad de un periodo viene influenciada por la volatilidad de los periodos inmediatamente anteriores;

b) existen factores no observables que también inciden en la volatilidad;

c) la muestra está compuesta por estimaciones de empresas que tienen distintos tamaños. Las variaciones en las empresas grandes son distintas a las variaciones de las empresas pequeñas;

d) cuando los analistas emiten sus estimaciones, los mercados reaccionan. A su vez, a la vista de esta reacción de los mercados los analistas ajustan sus estimaciones. Se establece así una circularidad analista-mercadoanalista. No hay que confundir este último ajuste con las revisiones que los analistas suelen realizar a sus estimaciones a la vista de nuevos análisis fundamentales.

Todas estas circunstancias pueden hacer que la medición de esa influencia del consenso de los analistas esté sesgada. Para evitar este sesgo se utiliza un modelo de regresión dinámica de panel, conocido también como modelo DPD (Arellano y Bond, 1991), que recoge la volatilidad de los meses anteriores, contrarresta el efecto de los factores no observables, neutraliza el efecto de la diferencia de tamaños de las empresas de la muestra y, en la circularidad analista-mercado-analista, aísla el efecto analista-mercado.

Si se hubiese utilizado un modelo de regresión lineal para datos de panel de efectos fijos o uno de efectos aleatorios se hubiera incorporado los sesgos señalados anteriormente, invalidando así la explicación del modelo.

Todos los modelos han sido sometidos a los oportunos contrastes para garantizar que los resultados sean significativos ${ }^{8}$.

El modelo de regresión dinámica con datos de panel (DPD), propuesto por Arellano and Bond (1991), tiene la siguiente especificación general:

\footnotetext{
${ }^{8}$ Estos contrastes son: Autocorrelación (Arellano-Bond), sobreidentificación (Sargan), estabilidad estructural del modelo (Chow) y significatividad conjunta (Wald).
} 
Ignacio Cervera Conte

$$
y_{i, t}=\delta+\sum_{l=1}^{L} \alpha_{l} y_{i, t-l}+\sum_{k=1}^{K} \beta_{k} x_{k, i, t}+\mu_{i}+\varepsilon_{i, t}
$$

Donde

$\delta$ es la constante.

$i$ se refiere a la empresa; toma valores $1 \ldots \mathrm{N}$.

$t$ se refiere al mes; toma valores $1 \ldots \mathrm{T}$.

$l$ se refiere al retardo de la variable dependiente; toma valores $1 \ldots \mathrm{L}$.

$k$ se refiere al número de variables explicativas; toma valores $1 . . . \mathrm{K}$.

$\alpha_{l}$ es el coeficiente de la variable dependiente retardada $l$ periodos.

$\chi_{k, i, t}$ es la observación de la empresa $i$ en el mes $t$ para la variable $k$.

$\beta_{k}$ es el coeficiente de la variable explicativa $k$.

$\mu_{i}$ recoge los efectos no observables que difieren entre las empresas

pero no en el tiempo.

$\varepsilon_{i, t}$ se refiere al término de error puramente aleatorio.

Este modelo utiliza un estimador GMM con las siguientes características:

$1^{a}$ Estimador en diferencia: se utiliza un estimador en diferencia ante la posibilidad de que factores no observables que varían en el tiempo estén correlacionados.

$2^{a}$ Estimador en dos etapas: Las relaciones entre los distintos agentes que intervienen en los mercados financieros tienen naturaleza dinámica. Es el caso de los analistas financieros; éstos publican sus pronósticos que pronto tienen repercusión en los mercados; pero tras ver esas repercusiones vuelven a revisar sus pronósticos. Este proceso tiene su concreción estadística en la presencia de una variable endógena retardada incluida entre las variables explicativas. Las regresiones en dos etapas son utilizadas para corregir problemas de endogeneidad. En la primera etapa, se estima el modelo para intentar explicar la variable que causa la endogeneidad; este modelo es utilizado en la segunda etapa para estimar un modelo sin endogeneidad.

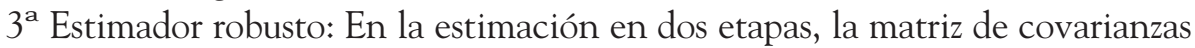
es ya robusta, pero típicamente produce errores estándar que son sesgados, especialmente en muestras finitas. Windemeijer (2005) propone una corrección para el problema del sesgo generado cuando se utilizan muestras finitas en la estimación. Para ello, en este modelo se utilizan errores estándar robustos para tomar en consideración la posible presencia de heterocedasticidad en los residuos del modelo.

\subsection{Variable dependiente referida a la reacción del mercado}

Los datos de este trabajo contienen la evolución de los precios de cierre de las acciones de las empresas que se están analizando así como la volatilidad de sus 
rendimientos. Los datos de los precios de cierre han sido facilitados por Infobolsa. Con estos datos se calcula los rendimientos de los precios que se definen como la variación porcentual del logaritmo neperiano del precio de cierre del valor para dos días consecutivos de mercado.

A partir de estos datos se calcula la volatilidad, como variable dependiente que recoge el impacto en el mercado de los datos de los analistas (González y Gimeno, 2008). Las series de carácter financiero presentan, entre otros aspectos, un agrupamiento y una persistencia de la volatilidad (García Centeno y Calvo Martín, 2005). Las series presentan un agrupamiento de la volatilidad, es decir, una volatilidad cambiante a lo largo del tiempo, alternando precios de baja volatilidad con precios de alta volatilidad. También se da una persistencia de la volatilidad, es decir, una repercusión del cambio en los precios que no desaparece inmediatamente sino que está presente varios periodos; y esto tiene su concreción estadística en una correlación en los cuadrados de los rendimientos de la serie que decrecen de forma lenta hacia cero. Con objeto de recoger estos aspectos, la volatilidad ha sido calculada siguiendo el modelo $\mathrm{GARCH}^{9}$ (Bollerslev, 1986), que actúa como un proceso adaptativo que tiene en cuenta la varianza condicionada en cada etapa, de forma que pueda agrupar conglomerados de alta volatilidad. Esta es una de las razones por las que este modelo tiene gran aplicación en el campo financiero. Con ello, la volatilidad calculada con el modelo GARCH es una volatilidad que mira más al futuro que la volatilidad clásica, demasiado dependiente del pasado.

Un modelo de Volatilidad GARCH $(1,1)$ tiene la siguiente forma (Bollerslev, 1986; Rodríguez Pinzón, 2009):

1) Ecuación de la media:

$$
\begin{aligned}
& \text { Rendimiento }_{i, t}=\left(\text { Volatilidadld }_{i, t}\right)^{1 / 2} \cdot \mathrm{u}_{i, t} \\
& \text { siendo } \quad \mathrm{u}_{i, t} \sim N(0,1)
\end{aligned}
$$

Lo que dice este modelo es que los rendimientos de cada día dependen de la volatilidad diaria que haya (que mide la intensidad del movimiento), mientras que la dirección del mismo (subida o bajada) depende de la variable aleatoria.

2) Ecuación de la varianza:

$$
\text { Volatilidadld }_{i, t}=\alpha_{0}+\alpha_{1} \cdot \text { Volatilidadld }_{i, t-1}+\beta \cdot\left(\text { Rendimiento }_{i, t-1}\right)^{2}
$$

${ }^{9}$ Generalized Autorregresive Condicional Heterocedastic (Heterocedasticidad Condicional Autorregresiva Generalizada). 
Ignacio Cervera Conte

siendo $\quad \alpha_{0}>0, \quad \alpha_{1}, \beta \geq 0, \quad \alpha_{1}+\beta<1$

Es decir, la volatilidad no es constante sino que va cambiando. La volatilidad no sólo cambia sino que persiste. En este modelo, la persistencia de la volatilidad viene dada por la suma $\alpha_{1}+\beta$.

Básicamente, la volatilidad que se tiene un día depende de la que hubo el día anterior, (que se aproxima mediante el rendimiento al cuadrado). Lo único que se necesita saber para tener la volatilidad de un día son dos cosas:

- Cuánto valen los parámetros $\alpha_{0}, \alpha_{1}$ y $\beta$ que se estiman con toda la muestra.

- Cuál fue el rendimiento que se produjo el día anterior.

Calculada la volatilidad diaria, se proyecta la misma de forma mensual.

\subsection{Variables explicativas referidas al consenso de los analistas}

El modelo pretende capturar la lógica del informe de los analistas (en forma de consenso) con sus tres núcleos: estimación de beneficios, cálculo del precio objetivo y la recomendación de la inversión. Por eso los datos de este trabajo contienen el consenso de las estimaciones de beneficios, precios objetivos y recomendaciones de los analistas financieros procedentes de la base de datos FactSet. El consenso de los analistas suministrado por FactSet para una empresa concreta y para un determinado mes es definido como la mediana de las estimaciones de los brokers realizadas en los últimos 100 días antes del final de cada mes. Este método utiliza las últimas técnicas para el cálculo del consenso (Wieland, 2011; Jegadeesh and Kim, 2010).

En la literatura previa sobre la actividad de los analistas financieros se han utilizado las bases de datos de First Call, Zack Investment Research e I/B/E/S. Ninguna de ellas se puede usar en España (López Espinosa y Gómez Sala, 2005). Las dos primeras no disponen de datos sobre recomendaciones de las sociedades cotizadas en el mercado español, mientras que $\mathrm{I} / \mathrm{B} / \mathrm{E} / \mathrm{S}$ contiene predicciones de beneficios pero no de recomendaciones de inversión.

Además, las investigaciones previas concluyen que las revisiones de las estimaciones tienen más influencia en el mercado que el valor de las estimaciones. Por eso, las variables explicativas serán las revisiones de las estimaciones. Los datos facilitados por FactSet se agrupan en torno a los siguientes conjuntos de variables $^{10}$ :

a) Revisiones de las estimaciones de consenso de beneficios por acción en cada mes: revisiones al alza (RevsubeEBPA1, RevsubeEBPA2), número de revisio-

\footnotetext{
${ }^{10}$ En paréntesis y cursiva aparecen las variables tal y como han sido nombradas en el trabajo empírico.
} 
nes a la baja (RevbajaEBPA1, RevbajaEBPA2), y número de revisiones sin cambios (RevigualEBPA1, RevigualEBPA2) de la estimación de los beneficios por acción para el final del año en curso (variables terminadas en 1) y para el final del año siguiente (variables terminadas en 2). Por ejemplo: al 31/01/2002, número de revisiones de la estimación de consenso de beneficios por acción para el 31/12/2002 y para el 31/12/2003.

b) Revisiones de los precios objetivos: número de revisiones del precio objetivo al alza (RevsubePO), número de revisiones a la baja (RevbajaPO) y número de revisiones sin cambios (RevigualPO).

c) Revisiones de las recomendaciones: número de revisiones al alza (RevsubeREC), a la baja (RevbajaREC) y sin cambio (RevigualREC). Considerando las recomendaciones de venta como el punto inferior de la escala de recomendaciones, los sucesivos cambios hacia las siguientes recomendaciones son considerados como revisiones al alza. Cuando el sentido de los cambios en la escala de recomendaciones es el contrario, entonces se consideran revisiones a la baja.

Para seleccionar una variable de cada grupo se procede a la satisfacción de tres criterios (procedimiento ilustrado en la figura del Anexo):

$1^{\circ}$ Que la variable explicativa seleccionada tenga una alta correlación con la variable dependiente (Volatilidad). Con ello se busca asegurar la relación 'volatilidad-variable explicativa' que pretende recoger el modelo.

$2^{\circ}$ Que dicha variable supere el test de causalidad de Granger para asegurar la causalidad estadística con respecto a la variable dependiente. Es decir, que asegure la causa-efecto de la relación que pretende recoger el modelo. Es decir, asegurar que es la variable la que explica la volatilidad y no al revés.

$3^{\circ}$ Que la variable explicativa seleccionada tenga una baja correlación con respecto a las otras variables explicativas, para evitar la multicolinealidad. En este sentido, se ofrecerá el número de condición de la matriz (condition index) para mostrar la no multicolinealidad. Con ello, se pretende asegurar la mayor independencia entre las variables que van a explicar la volatilidad y así aislar el efecto de cada variable explicativa sobre la variable dependiente.

Las variables que superan estos criterios son: número de revisiones a la baja del precio objetivo (RevbajaPO), número de revisiones sin cambios de la estimación de los beneficios por acción para el final del año siguiente (RevigualEBPA2) y número de revisiones que elevan el grado de recomendación (RevsubeREC). 


\subsection{Estadística descriptiva}

A continuación en la tabla 2 se muestra la estadística descriptiva de las variables explicativas:

\section{Tabla 2. Estadística descriptiva}

Esta tabla informa de la estadística de las variables explicativas: RevbajaPO es el número de revisiones mensuales a la baja del Precio Objetivo de consenso; RevigualEBPA2 es el número de revisiones mensuales que no modifica la estimación de consenso del BPA para el final del año siguiente; RevsubeREC es el número de revisiones mensuales que eleva el grado de Recomendación de consenso (se eleva el grado de Recomendación cuando va en la dirección vender-mantener-comprar).

\begin{tabular}{|c|c|c|c|c|c|c|}
\hline Año & Variable & $\mathrm{N}$ & Media & $\begin{array}{c}\text { Desviación } \\
\text { Típica }\end{array}$ & $\begin{array}{l}\text { Coeficiente } \\
\text { de variación }\end{array}$ & Mediana \\
\hline 2002 & $\begin{array}{l}\text { RevbajaPO } \\
\text { RevigualEBPA2 } \\
\text { RevsubeREC }\end{array}$ & $\begin{array}{l}600 \\
599 \\
600\end{array}$ & $\begin{array}{l}1,21 * * * \\
3,40 * * * \\
1,36 * * *\end{array}$ & $\begin{array}{l}1,86 \\
3,18 \\
1,65\end{array}$ & $\begin{array}{l}1,54 \\
0,94 \\
1,22\end{array}$ & $\begin{array}{l}0 \\
3 \\
1\end{array}$ \\
\hline 2003 & $\begin{array}{l}\text { RevbajaPO } \\
\text { RevigualEBPA2 } \\
\text { RevsubeREC }\end{array}$ & $\begin{array}{l}600 \\
600 \\
600\end{array}$ & $\begin{array}{l}1,07 * * * \\
4,17 * * * \\
1,39 * * *\end{array}$ & $\begin{array}{l}1,63 \\
3,72 \\
1,79\end{array}$ & $\begin{array}{l}1,53 \\
0,89 \\
1,29\end{array}$ & $\begin{array}{l}1 \\
3 \\
1\end{array}$ \\
\hline 2004 & $\begin{array}{l}\text { RevbajaPO } \\
\text { RevigualEBPA2 } \\
\text { RevsubeREC }\end{array}$ & $\begin{array}{l}600 \\
600 \\
600\end{array}$ & $\begin{array}{l}0,78 * * * \\
5,43 * * * \\
1,25 * * *\end{array}$ & $\begin{array}{l}1,17 \\
4,32 \\
1,65\end{array}$ & $\begin{array}{l}1,51 \\
0,79 \\
1,31\end{array}$ & $\begin{array}{l}0 \\
4 \\
1\end{array}$ \\
\hline 2005 & $\begin{array}{l}\text { RevbajaPO } \\
\text { RevigualEBPA2 } \\
\text { RevsubeREC }\end{array}$ & $\begin{array}{l}600 \\
593 \\
600\end{array}$ & $\begin{array}{l}0,63 * * * \\
3,88 * * * \\
1,09 * * *\end{array}$ & $\begin{array}{l}1,20 \\
3,75 \\
1,43\end{array}$ & $\begin{array}{l}1,91 \\
0,97 \\
1,31\end{array}$ & $\begin{array}{l}0 \\
3 \\
1\end{array}$ \\
\hline 2006 & $\begin{array}{l}\text { RevbajaPO } \\
\text { RevigualEBPA2 } \\
\text { RevsubeREC }\end{array}$ & $\begin{array}{l}600 \\
594 \\
600\end{array}$ & $\begin{array}{l}0,78 * * * \\
3,60 * * * \\
1,19 * * *\end{array}$ & $\begin{array}{l}2,10 \\
3,14 \\
1,52\end{array}$ & $\begin{array}{l}2,70 \\
0,87 \\
1,28\end{array}$ & $\begin{array}{l}0 \\
3 \\
1\end{array}$ \\
\hline 2007 & $\begin{array}{l}\text { RevbajaPO } \\
\text { RevigualEBPA2 } \\
\text { RevsubeREC }\end{array}$ & $\begin{array}{l}600 \\
588 \\
600\end{array}$ & $\begin{array}{l}1,12 * * * \\
3,70 * * * \\
1,31 * * *\end{array}$ & $\begin{array}{l}1,79 \\
3,30 \\
1,67\end{array}$ & $\begin{array}{l}1,60 \\
0,89 \\
1,27\end{array}$ & $\begin{array}{l}0 \\
3 \\
1\end{array}$ \\
\hline 2008 & $\begin{array}{l}\text { RevbajaPO } \\
\text { RevigualEBPA2 } \\
\text { RevsubeREC }\end{array}$ & $\begin{array}{l}600 \\
600 \\
600\end{array}$ & $\begin{array}{l}4,29 * * * \\
3,28 * * * \\
0,98 * * *\end{array}$ & $\begin{array}{l}4,21 \\
3,02 \\
1,59\end{array}$ & $\begin{array}{l}0,98 \\
0,92 \\
1,62\end{array}$ & $\begin{array}{l}3 \\
3 \\
0\end{array}$ \\
\hline 2009 & $\begin{array}{l}\text { RevbajaPO } \\
\text { RevigualEBPA2 } \\
\text { RevsubeREC }\end{array}$ & $\begin{array}{l}600 \\
600 \\
600\end{array}$ & $\begin{array}{l}2,80 * * * \\
3,36 * * * \\
1,05 * * *\end{array}$ & $\begin{array}{l}3,51 \\
3,08 \\
1,61\end{array}$ & $\begin{array}{l}1,26 \\
0,92 \\
1,54\end{array}$ & $\begin{array}{l}2 \\
3 \\
0\end{array}$ \\
\hline Total & $\begin{array}{l}\text { RevbajaPO } \\
\text { RevigualEBPA2 } \\
\text { RevsubeREC }\end{array}$ & $\begin{array}{l}4800 \\
4774 \\
4800\end{array}$ & $\begin{array}{l}1,58 * * * \\
2,97 * * * \\
1,20 * * *\end{array}$ & $\begin{array}{l}2,70 \\
3,69 \\
1,62\end{array}$ & $\begin{array}{l}1,70 \\
1,24 \\
1,35\end{array}$ & $\begin{array}{l}1 \\
2 \\
1\end{array}$ \\
\hline
\end{tabular}

*** nivel de significación de la media $(\mathrm{p}<0,001)$ 
Se puede observar que el número de revisiones a la baja del precio objetivo aumenta a partir del comienzo de la crisis, lo cual tiene sentido ya que este precio es el resultado de descontar los futuros flujos de caja libre de la empresa que están analizando. Si los flujos futuros disminuyen y el tipo de descuento aumenta para recoger el mayor riesgo, los precios objetivos se ven abocados a una caída. Al mismo tiempo existe una disminución en las otras dos variables con el paso del tiempo y según se va acercando el comienzo de la crisis.

\subsection{Contrastes de hipótesis en el modelo}

El modelo es sometido a los siguientes contrastes:

\section{1) Contraste de autocorrelación (Arellano-Bond)}

Como ya se ha viso previamente, en las regresiones de paneles dinámicos se considera la variable dependiente retardada como variable explicativa, lo cual explica la presencia de autocorrelación. La estimación por GMM se realiza en dos etapas y permite usar en la segunda etapa una matriz de ponderaciones elaborada con los errores de la primera etapa. Es preciso contrastar que no existe autocorrelación de segundo orden porque el estimador GMM se basa en la restricción de momentos (condición de ortogonalidad).

$$
E\left(\varepsilon_{i, t} \varepsilon_{i, t-2}\right)=0
$$

Para contrastar la consistencia de las estimaciones, se presenta el contraste de ausencia de correlación serial de segundo orden propuesto por Arellano y Bond (1991). El contraste estadístico de Arellano-Bond, permite contrastar esta hipótesis y se distribuye asintóticamente como una normal estandarizada $N(0,1)$ bajo la hipótesis nula de no correlación serial. Si la hipótesis nula no es rechazada, las estimaciones son consistentes desde este criterio.

\section{2) Contraste de la validez de las variables instrumentales (Sargan)}

El modelo DPD utiliza variables instrumentales. Una variable instrumental debe satisfacer dos requisitos: a) estar incorrelacionada con el error y b) estar correlacionada con la variable explicativa endógena que instrumentaliza (Wooldridge, 2006). Se suele realizar un contraste de sobreidentificación para validar la utilización de los instrumentos adicionales. Este es el contraste de Sargan: un contraste de sobreidentificación de las restricciones, asintóticamente distribuido como una con tantos grados de libertad como número de restricciones de sobrei- 
dentificación se contrastan (Sargan, 1964). La hipótesis que se contrasta es que las variables instrumentales no están correlacionadas con algún grupo de errores, y por consiguiente son aceptables como instrumentos. Si la hipótesis nula no es rechazada, los instrumentos pasan el contraste y son declarados válidos desde este criterio.

\section{3) Presencia de heterocedasticidad}

En la estimación en dos etapas, la matriz de covarianzas es ya robusta, pero típicamente produce errores estándar que son sesgados, especialmente en muestras finitas. Como se ha indicado previamente, este modelo utiliza errores estándar robustos para tomar en consideración la posible presencia de heterocedasticidad en los residuos del modelo.

\section{4) Contraste de significatividad conjunta de las variables (Wald)}

Se emplea con el objetivo de comprobar la significatividad estadística conjunta de los parámetros estimados. Este estadístico se distribuye asintóticamente como una $X^{2}$ con un número de grados de libertad igual al número de coeficientes estimados, bajo la hipótesis nula de no significación:

$$
\mathrm{H}_{0}: \beta_{1}=\beta_{2}=\ldots=\beta_{\mathrm{K}}=0 \text {, }
$$

donde para $\mathrm{K}$ variables $\beta_{k}$ explicativas es el parámetro de la variable explicativa $X_{k}$.

Si la hipótesis nula es rechazada, entonces el conjunto de las variables es significativo y, por consiguiente, se aceptan.

\section{5) Contraste de la estabilidad estructural del modelo (Chow)}

Como cualquier modelo econométrico, el modelo aquí presentado establece como hipótesis de partida que los parámetros asociados a cada variable explicativa son únicos y válidos para representar la relación entre la variable dependiente y cada una de las variables explicativas en la muestra de datos seleccionada. Esto es, los parámetros asociados a cada variable explicativa son idénticos para todas las muestras analizadas, y por lo tanto la estructura de relaciones entre variables se mantiene estable. No hay, por consiguiente, un cambio estructural en los parámetros entre los periodos analizados. A partir de un análisis de los errores estándar de las dos estimaciones por separado y otra tercera uniendo ambas muestras, el contraste de Chow (1960) permite verificar si se dio cambio estructural en los parámetros. En concreto, el contraste de Chow va a permitir 
justificar estadísticamente un modelo para cada subperíodo de la muestra, de los que luego se hablará.

A partir de los parámetros del modelo (1), el contraste de Chow contrasta la hipótesis,

$$
\mathrm{H}_{0}: \beta_{k}^{P R C}=\beta_{k}^{C R}
$$

donde

$$
\begin{gathered}
P R C=\text { Etapa Pre-Crisis } \\
C R=\text { Etapa Crisis }
\end{gathered}
$$

Si se rechaza la hipótesis, se admite la distinción estadística entre los subperiodos, justificando un modelo para cada uno de ellos.

\section{RESULTADOS: LA CONTRIBUCIÓN DE LOS ANALISTAS}

La tabla recoge el resultado de los modelos. El contraste de Chow arrojó el siguiente resultado:

$$
\begin{gathered}
\chi^{2}(8)=46,84 \\
\text { Prob }>\chi^{2}=0,000
\end{gathered}
$$

Es decir, los coeficientes de las variables del Mercado Continuo de la etapa pre-crisis no coinciden con los de las variables de la etapa de crisis y, por consiguiente, para este mercado tiene sentido realizar un modelo para cada etapa dentro del mismo periodo. 
Tabla 3. Modelos DPD

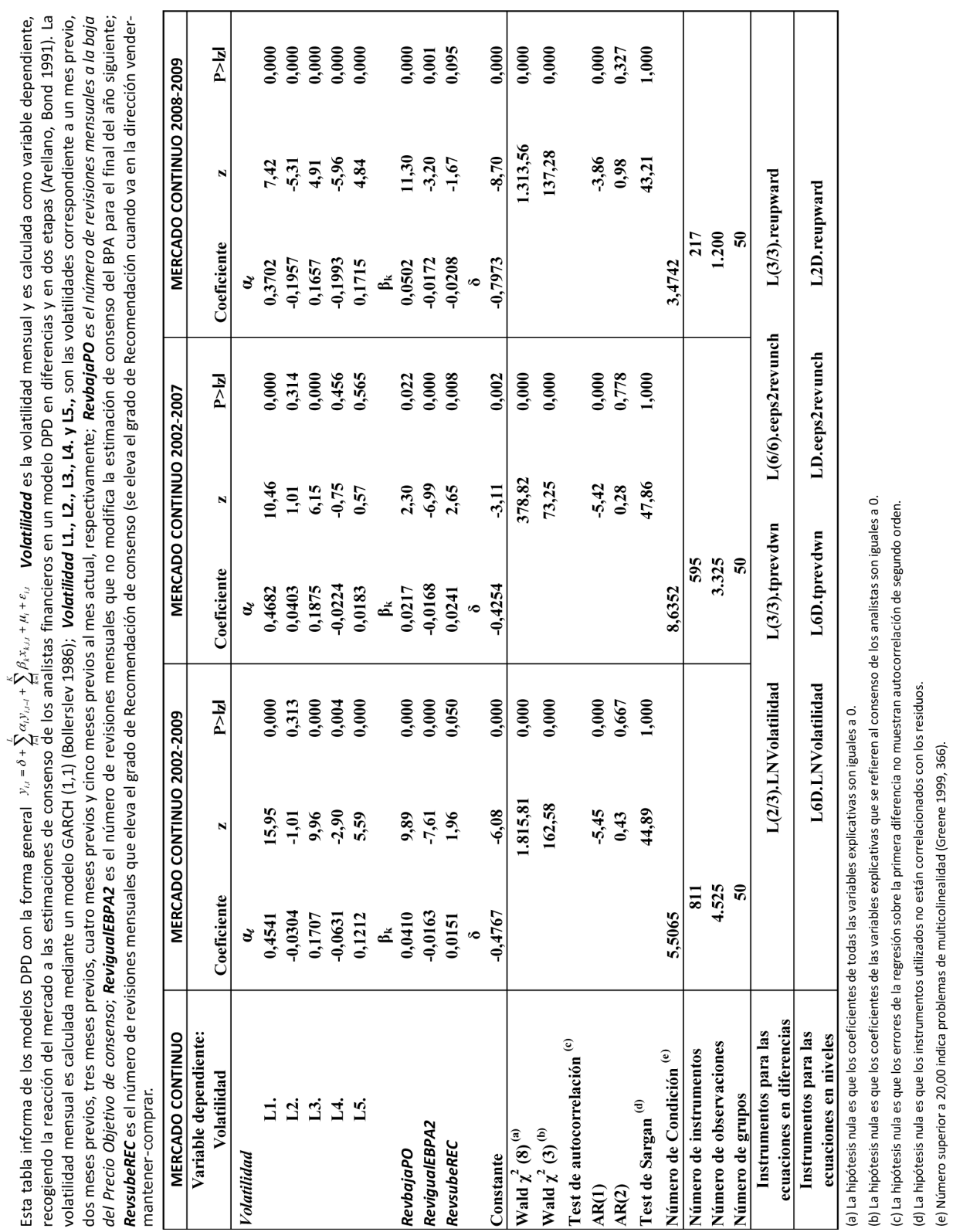

Para poder determinar la influencia de cada variable se procede a su estandarización con su media y su desviación típica. El resultado aparece en la tabla 4: 


\section{Tabla 4. Influencia de las variables que explican la volatilidad}

Esta tabla informa de los modelos DPD con las variables estandarizadas con el fin de reflejar la contribución de cada variable. Volatilidad es la volatilidad mensual y es calculada como variable dependiente, recogiendo la reacción del mercado a las estimaciones de consenso de los analistas financieros en un modelo DPD en diferencias y en dos etapas (Arellano, Bond 1991). La volatilidad mensual es calculada mediante un modelo GARCH $(1,1)$ (Bollerslev 1986); Volatilidad L1., L2., L3., L4. y L5., son las volatilidades correspondiente a un mes previo, dos meses previos, tres meses previos, cuatro meses previos y cinco meses previos al mes actual, respectivamente; RevbajaPO es el número de revisiones mensuales a la baja del Precio Objetivo de consenso; RevigualEBPA2 es el número de revisiones mensuales que no modifica la estimación de consenso del BPA para el final del año siguiente; RevsubeREC es el número de revisiones mensuales que eleva el grado de Recomendación de consenso (se eleva el grado de Recomendación cuando va en la dirección: vender-mantener-comprar).

\begin{tabular}{|c|c|c|c|}
\hline Variable dependiente: Volatilidad & \multicolumn{3}{|c|}{ MERCADO CONTINUO } \\
\hline Variables explicativas & $2002-2009$ & $2002-2007$ & $2008-2009$ \\
\hline Volatlidad* & & & \\
\hline L1. & 0,520 & 0,375 & 0,429 \\
\hline L2. & $-0,054$ & 0,040 & $-0,155$ \\
\hline L3. & 0,111 & 0,115 & 0,100 \\
\hline L4. & $-0,078$ & $-0,118$ & $-0,092$ \\
\hline L5. & 0,105 & 0,134 & $-0,034$ \\
\hline RevbajaPO* & 0,230 & 0,067 & 0,381 \\
\hline RevigualEBPA2* & $-0,134$ & $-0,121$ & $-0,144$ \\
\hline RevsubeREC* & 0,037 & 0,038 & $-0,067$ \\
\hline Constante & 0,003 & $-0,010$ & 0,024 \\
\hline
\end{tabular}

* Variable normalizada con su media y desviación típica

Entre las variables que forman el consenso de los analistas, la que corresponde al número de revisiones a la baja del precio objetivo es la más influyente (en valor absoluto) en la volatilidad. Durante la crisis se acentúa la influencia de las revisiones a la baja de los precios objetivos en la volatilidad. Si los inversores se muestran sensibles a las revisiones que corrigen a la baja los precios objetivos, esta sensibilidad se ve considerablemente aumentada en periodos donde las cotizaciones bursátiles son descendentes.

La siguiente variable más influyente (en valor absoluto) de las que reflejan el consenso de los analistas es la que corresponde a las revisiones que dejan intactas las previsiones de los BPA (beneficio por acción) del año siguiente. Las revisiones que mantienen los BPA esperados para el año siguiente reducen la volatilidad, por eso aparece con signo negativo en todos los modelos. Lo cual tiene sentido porque hay más incertidumbre en el año siguiente que en el año en curso y si las revisiones confirman los beneficios anteriormente estimados, la incertidumbre se reduce. La influencia de esa confirmación es más grande en la crisis. 
Por último, la variable que menos influencia muestra es la que se refiere a las revisiones que elevan el grado de la recomendación. Según estos modelos, los inversores prestan más atención a la información contenida en el precio objetivo y en las estimaciones de los beneficios, sin rechazar la recomendación. ¿Qué valor tiene entonces la recomendación a la vista de los anteriores datos? La recomendación evidencia el grado de convicción de los analistas con respecto a sus propias estimaciones y sus revisiones.

Ya se puede ver que en la volatilidad de los mercados bursátiles intervienen muchos factores. La volatilidad de un periodo viene explicada inicialmente por la volatilidad de los periodos anteriores; pero además intervienen otros factores, muchos de los cuales no son observables y, a priori, desconocidos. La información del consenso de los analistas ayuda a identificar algunos de esos factores subyacentes en los cambios de la volatilidad, reduciendo así los factores desconocidos.

Tabla 5. Desviación típica de los errores residuales

\begin{tabular}{|c|c|c|c|}
\hline \multirow{2}{*}{ MODELOS } & \multicolumn{3}{|c|}{ NÚMERO DE BROKERS } \\
\cline { 2 - 4 } & $2002-2009$ & $2002-2007$ & $2008-2009$ \\
\hline Con Analistas & 0,082687 & 0,071773 & 0,108144 \\
Sin Analistas & 0,098769 & 0,077859 & 0,131310 \\
\hline Diferencia & $-0,016082$ & $-0,006086$ & $-0,023166$ \\
\hline Diferencia (\%) & $-16,28 \%$ & $-7,82 \%$ & $-17,64 \%$ \\
\hline
\end{tabular}

La tabla 5 nos informa que con el consenso de los analistas se conocen más factores que influyen en la volatilidad. Por consiguiente, el consenso nos ofrece más información acerca de la volatilidad de los mercados y de los factores que intervienen en su evolución.

Se puede observar también que, en las etapas de crisis, el consenso de los analistas duplica su capacidad explicativa con respecto a la etapa pre-crisis. Los inversores prestan más atención a las estimaciones de los analistas en épocas de tendencia bursátil descendente que en época de cotizaciones ascendentes. La incertidumbre que caracteriza a la etapa de crisis mueve a los inversores a buscar más información en los pronósticos de los analistas de valores.

Para concretar aún más la influencia de los analistas en la evolución de la volatilidad se procede al cálculo del coeficiente de determinación $\left(\mathrm{R}^{2}\right)$ de los modelos con las variables que se refiere a los analistas y de los modelos sin la presencia de estas variables. 
La diferencia entre los coeficientes de determinación de los modelos con analistas y los modelos sin analistas señala el porcentaje de la volatilidad explicada por el consenso de los analistas, tal y como se recoge en la tabla 6:

\section{Tabla 6. Porcentaje de la volatilidad explicada por el consenso de los analistas (R2)}

\begin{tabular}{|l|r|r|r|}
\hline \multirow{2}{*}{} & \multicolumn{2}{|c|}{ MERCADO CONTINUO } \\
\cline { 2 - 4 } & $2002-2009$ & $2002-2007$ & $2008-2009$ \\
\hline (A) R de los Modelos sin Consenso de los & $\begin{array}{r}94,12 \% \\
\text { Analistas }\end{array}$ & $\begin{array}{r}96,35 \% \\
3,65 \%\end{array}$ & $\begin{array}{r}89,61 \% \\
10,39 \%\end{array}$ \\
(B) Varianza Inexplicada & $95,88 \%$ & $96,90 \%$ & $92,96 \%$ \\
\hline (C) R de los Modelos con Consenso de los & $1,76 \%$ & $0,55 \%$ & $3,34 \%$ \\
$\begin{array}{l}\text { Analistas } \\
\text { (D) Diferencia entre Modelos (C)-(A) }\end{array}$ & $29,91 \%$ & $15,02 \%$ & $32,17 \%$ \\
\hline \% de la Varianza Inexplicada explicada por & & & \\
el Consenso de los Analistas (D):(B) & & & \\
\hline
\end{tabular}

Aún tratándose de una influencia modesta en términos cuantitativos, se trata sin embargo de una influencia significativa. La influencia del consenso de los analistas en la volatilidad es más grande en la etapa de la crisis que en la etapa previa. Dicho consenso explica el 3,34\% de la variación de la volatilidad en el Mercado Continuo en la etapa de la crisis en dicho mercado, lo que representa un $32,17 \%$ de la varianza no explicada sin la presencia del consenso de los analistas. Lo que sigue confirmando los anteriores resultados: el consenso de los analistas financieros ejerce una mayor influencia sobre la volatilidad bursátil en la etapa de la crisis que en la etapa previa a la crisis.

\section{INTERPRETACIÓN DE LA CONTRIBUCIÓN DE LOS ANALISTAS}

El modelo no asume necesariamente que todas las variables tomen valores simultáneamente cada mes, aunque podría darse casos como en el siguiente: los precios objetivos son calculados descontando los flujos de caja libres esperados en los próximos años, siendo los beneficios estimados uno de los componentes de esos flujos; así los precios objetivos pueden variar sin que varíen los beneficios estimados, haciéndolo las inversiones en capital y las necesidades operativas de financiación (las otras componentes de los flujos de caja).

Llegados a este punto, ¿cómo interpretar el efecto de cada variable explicativa en la volatilidad? 


\subsection{Las revisiones de los precios objetivos}

Es sin duda las revisiones que mayor influencia ejercen sobre la volatilidad, si se compara con las revisiones de los beneficios y las revisiones del grado de recomendación. Este hecho es comprensible al intervenir en la elaboración del precio objetivo un espectro más amplio de información que el utilizado para las estimaciones de beneficios. Ya se ha recordado que la literatura financiera ha atestiguado un sesgo optimista persistente en las estimaciones de los analistas. Conocido este sesgo, una revisión a la baja tiene más impacto sobre la volatilidad que una revisión al alza por resultar aquélla más inesperada. Los inversores saben que cuando los analistas bajan el precio objetivo tras una revisión del mismo lo hacen porque ha aparecido una información nueva no esperada por aquéllos.

La crisis financiera ha acentuado este efecto. Cuando el consenso de los analistas incorpora un evento inesperado, esta incorporación se deja sentir en la volatilidad, muy especialmente en época de crisis financiera.

\subsection{Las revisiones de los beneficios estimados}

Sobre los beneficios estimados para el final del año en curso pesan menos incertidumbres que sobre los beneficios estimados para el año siguiente. Es fácil concluir que el consenso de estos últimos tiene más repercusión en la volatilidad. Cuando se revisan los beneficios esperados para el ejercicio siguiente y dichas revisiones no alteran las estimaciones realizadas con anterioridad, resulta lógico pensar que esta confirmación reduzca la volatilidad. Los analistas, a través de las revisiones, van actualizando sus estimaciones con información nueva. Cuando las revisiones dejan intactos los beneficios esperados para el año siguiente, se reduce el grado de incertidumbre.

En la etapa de crisis la mayor incertidumbre empuja al inversor a cerciorarse con más insistencia de las expectativas con las que está tomando sus decisiones. Uno de los canales por donde le llega esta confirmación es el consenso de los analistas.

\subsection{Las revisiones de las recomendaciones}

El consenso mostrado en las revisiones de las recomendaciones es el que menos influencia ejerce sobre la volatilidad. Si las recomendaciones fueran decisivas para los inversores, las estimaciones de los beneficios futuros y del precio objetivo no tendrían la relevancia mostrada anteriormente. Los inversores se fijan en la recomendación, pero se fijan antes en las estimaciones que han llevado al analista a formular su recomendación, porque encuentran en esas estimaciones más contenido informativo para sus decisiones, que en la propia recomendación. Al mismo tiempo, ante una misma recomendación, no todos los inversores actúan en la dirección marcada por la misma, ya que no todos los inversores parten de 
carteras con las mismas ponderaciones en sus títulos. ¿Qué efecto tiene pues la recomendación y sus revisiones? La literatura financiera (Stickel, 1995; Jegadeesh et al. 2004) ha confirmado que la recomendación aporta al inversor el grado de convicción con que los analistas muestran sus estimaciones.

En la presente investigación se ha visto que el efecto que ejerce sobre la volatilidad las revisiones que elevan el grado de recomendación tiene distintos signos en la etapa precrisis y en la etapa de la crisis. Ante un volumen de transacciones bajos, como es el caso de la etapa precrisis, la recomendación de los analistas anima a aumentar el número de transacciones en el Mercado Continuo, aumentando la volatilidad. Sin embargo, en la etapa de crisis, con volatilidades ya altas, un cambio en la recomendación a compra o a sobreponderación tiene un efecto negativo sobre la volatilidad ya que impera más la cautela que la recomendación.

\section{CONCLUSIÓN}

El consenso de los analistas financieros tiene una influencia cuantitativamente pequeña pero significativa en la volatilidad de los mercados. Los inversores cuentan con esta información que completa a otras de cara a la toma de decisiones. Esta influencia del consenso aumenta claramente con la crisis. La razón de esta influencia se encuentra en su rol comunicador de la información y de las expectativas acerca de las firmas que analizan. Las expectativas sobre la evolución de las compañías que cotizan en el Mercado Continuo no son directamente observables por los agentes que intervienen en el mercado. El consenso de los analistas ayuda a visualizar esas expectativas, pero sabiendo que no todas las expectativas son capturadas por el consenso.

El consenso es un canal de información acerca de factores inesperados y, al mismo tiempo, es un vehículo que confirma algunas de las expectativas proyectadas por los analistas. En la crisis financiera la incertidumbre ha aumentado considerablemente. Este hecho hace que el papel del consenso de los analistas financieros sea algo más intenso.

Futuras investigaciones en otros mercados como el europeo o el americano podrían confirmar estos resultados y ver aspectos diferenciales con respecto al Mercado Continuo.

\section{BIBLIOGRAFÍA}

ABARBANELL, J. S.; LANEN, W. N. y VERRECCHIA, R. E. (1995), "Analysts' forecasts as proxies for investor beliefs in empirical research", Journal of Accounting and Economics, Vol. 20, núm. 1, pp. 31-60. 
AIOLFI, M.; RODRÍGUEZ, M. y TIMMERMANN, A. (2010), “Understanding Analysts' Earnings Expectations: Biases, Nonlinearities and Predictability", Journal of Financial Econometrics, Vol. 8, núm. 3, pp. 305-334.

ARELLANO, M. y BOND, S. (1991), "Some Tests of Specification for Panel Data: Monte Carlo Evidence and an Application to Employment Equations.", Review of Economic Studies, Vol. 58, núm, 194, pp. 277.

ASQUITH, P.; MIKHAIL, M. B. y AU, A. S. (2005), "Information content of equity analyst reports", Journal of Financial Economics, Vol. 75, núm. 2, pp. 245-282.

BRAV, A. y LEHAVY, R. (2003), “An Empirical Analysis of Analysts' Target Prices: Short-term Informativeness and Long-term Dynamics”, Journal of Finance, Vol. 58, núm. 5, pp. 1933-1968.

BOLLERSLEV, T. (1986), "Generalized autoregressive conditional heteroskedasticity”, Journal of Econometrics, Vol. 31, núm. 3, pp. 307-327.

CHOW, G. (1960), "Tests of Equality Between Sets of Coefficients in Two Linear Regressions", Econometrica, Vol. 28, núm. 3, pp. 591-605.

COWEN, A.; GROYSBERG, B. y HEALY, P. (2006), "Which types of analyst firms are more optimistic?", Journal of Accounting and Economics, Vol. 41, núm. 1-2, pp. 119-146.

FELDMAN, R.; LIVNAT, J. y ZHANG, Y. (2012), “Analysts' Earnings Forecast, Recommendation and Target Price Revisions", Journal of Portfolio Management, Vol. 38, núm. 3, pp. 120-132.

GARCÍA CENTENO, M. y CALVO MARTÍN, M. E. (2005), "Estimación de modelos de volatilidad estocástica en series de rendimientos bursátiles.", XIII Jornadas A Coruña 2005, Documento técnico, Asociación Española de Profesores Universitarios de Matemáticas para la Economía y la Empresa (Asepuma). GIMEIN, M. (16 Septiembre 2002), "Eliot Spitzer", Fortune Magazine, pp. 77-84.

GONZÁLEZ, C. I. Y GIMENO, R. (2008), "Financial Analysts Impact on Stock Volatility: A Study on the Pharmaceutical Sector", Catedra FEDEA-La Caixa, Serie Economía de la Salud y Hábitos, Working Paper, 2008-19, disponible en SSRN eLibrary: http://ssrn.com/paper=1132938 [consulta: 30/05/2013].

HIRST, E. D.; KOONCE, L. y SIMKO, P. J. (1995), "Investor Reactions to Financial Analysts' Research Reports.", Journal of Accounting Research, Vol. 33, núm. 2, pp. 335-351.

JEGADEESH, N; KIM, J; KRISCHE, S. D. y LEE, C. M. C (2004), “Analyzing the analysts: When Do Recommendations Add Value?", Journal of Finance, Vol. 59, núm. 3, pp. 1083-1124.

JEGADEESH, N. y KIM, W. (2010), "Do Analysts Herd? An Analysis of Recommendations and Market Reactions", Review of Financial Studies, Vol. 23, núm. 2, pp. 901-937. 
KERL, A. G. y WALTER, A. (2008), "Target Price Accuracy", The European Financial Management Association, EFMA2009, Working Paper, 37, disponible en:

http://www.efmaefm.org/OEFMAMEETINGS/EFMA\%20ANNUAL\%20 MEETINGS/2009-milan/37.pdf [consulta: 20/12/2012].

LARRÁN, M. y REES, W. (2000), "Los incentivos de los analistas financieros para pronosticar con sesgo optimistas", Revista de Contabilidad, Vol. 3, núm. 6, pp. 81-114.

LÓPEZ ESPINOSA, G. y GÓMEZ SALA, J. (2005), "El valor de las recomendaciones de consenso de los analistas financieros en el mercado de capitales español", Instituto Valenciano de Investigaciones Económicas, Working paper WP-EC 2005-09.

MAIELLO, M. (11 Diciembre 2000), "Moving Targets", Forbes.com. Disponible en: http://www.forbes.com/forbes/2000/1211/6615288a.html [consulta: $15 / 12 / 2012]$

MORGENSON, G. (5/8/2001), "Price Targets Are Hazardous to Investors" Wealth", The New York Time.

PENMAN, S. H. (2010), Financial Satement Analysis and Security Valuation, McGraw-Hill, New York.

RAMNATH, S.; ROCK, S. K. y SHANE, P. B. (2008), "The Financial Analyst Forecasting Literature: A Taxonomy with Suggestions for Further Research", International Journal of Forecasting, Vol. 24, núm. 1, pp. 34-75.

RODRÍGUEZ PINZÓN, H. Y. (2009), "Profundización teórica de modelos de volatilidad ARCH-GARCH y una aplicación al caso colombiano", Facultad de Estadística, Universidad Santo Tomás, Colombia.

SARGAN, J. (1964), "Wages and Prices in the United Kingdom: A study of Econometric Methodology", en P. E. Hart; G. Mills y J. K. Whitaker, ed., Econometric Analysis for National Economic Planning, Butterworth Co., London, UK, pp. 25-63.

SCHIPPER, K. (1991), "Analysts' Forecasts”, Accounting Horizons, núm. 4, pp. $105-121$.

STICKEL, S. E. (1995), "The Anatomy of the Performance of Buy and Sell Recommendations", Financial Analysts Journal, Vol. 51, núm. 5, pp. 25-39.

WIELAND, M. (2011), "Identifying Consensus Analysts' Earnings Forecasts that Correctly and Incorrectly Predict an Earnings Increase", Journal of Business Finance and Accounting, Vol. 38, núm. 5-6, pp. 574-600.

WINDMEIJER, F. (2005), "A finite sample correction for the variance of linear efficient two-step GMM estimators", Journal of Econometrics, Vol. 126, núm. 1, pp. 25-51.

WOOLDRIDGE, J. (2006), Introducción a la Econometría: Un Enfoque Moderno, Thomson Paraninfo, Madrid. 


\section{ANEXO}

Figura 1. Procedimiento de selección de las variables explicativas del modelo

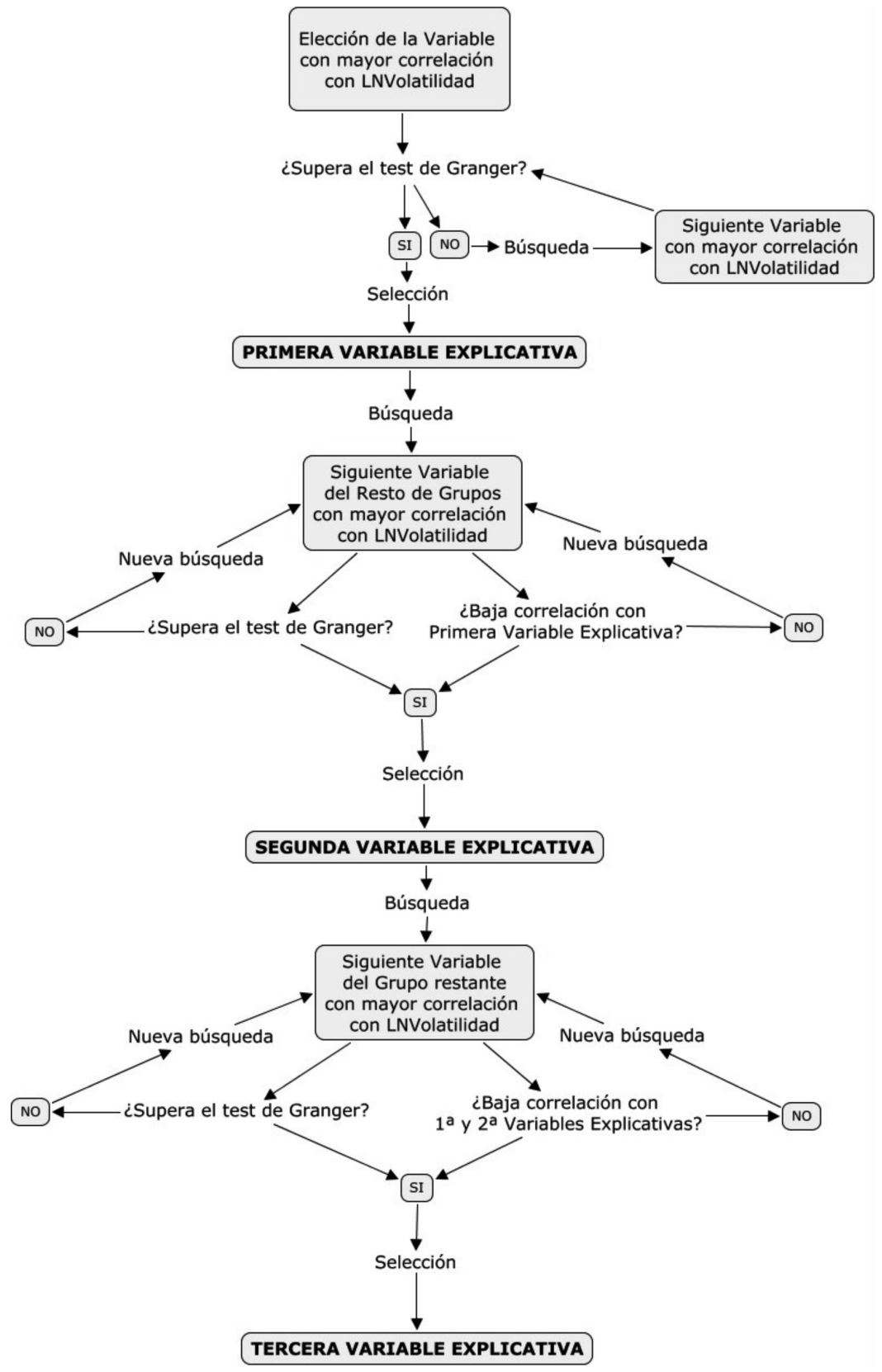

